\title{
\begin{tabular}{l|l} 
Mibraries & DSpace@MIT
\end{tabular}
}

\author{
MIT Open Access Articles
}

\section{Electrical Homogeneity of Large-Area Chemical Vapor Deposited Multilayer Hexagonal Boron Nitride Sheets}

The MIT Faculty has made this article openly available. Please share how this access benefits you. Your story matters.

Citation: Hui, Fei et al. "Electrical Homogeneity of Large-Area Chemical Vapor Deposited Multilayer Hexagonal Boron Nitride Sheets." ACS Applied Materials \& Interfaces 9, 46 (November 2017): 39895-39900 @ 2017 American Chemical Society

As Published: http://dx.doi.org/10.1021/ACSAMI.7B09417

Publisher: American Chemical Society (ACS)

Persistent URL: https://hdl.handle.net/1721.1/121494

Version: Original manuscript: author's manuscript prior to formal peer review

Terms of Use: Article is made available in accordance with the publisher's policy and may be subject to US copyright law. Please refer to the publisher's site for terms of use. 


\section{Electrical homogeneity of large-area chemical vapor}

\section{deposited multilayer hexagonal boron nitride sheets}

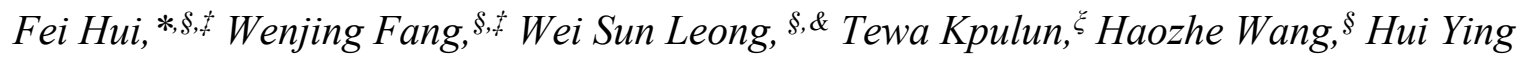
Yang, ${ }^{\&}$ Marco A. Villena,${ }^{*}$ Gary Harris,${ }^{\zeta}$ Jing Kong, ${ }^{\S}$ Mario Lanza*

*Institute of Functional Nano \& Soft Materials, Collaborative Innovation Center of Suzhou Nano Science and Technology, Soochow University, Suzhou, 215123, China.

${ }^{\S}$ Department of Electrical Engineering and Computer Science, Massachusetts Institute of Technology, Cambridge, MA 02139, USA.

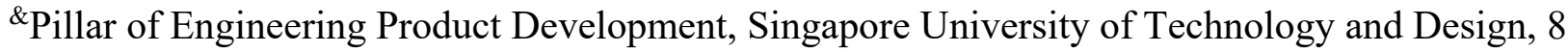
Somapah Road, Singapore 487372, Singapore

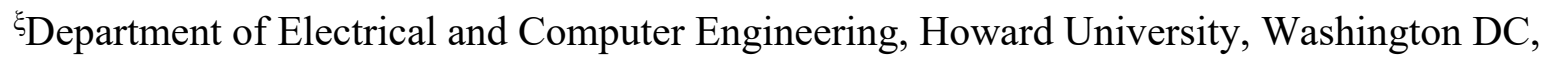
20059, USA.

${ }^{\delta}$ Department of Materials Science and Engineering, Stanford University, CA 94305, USA

KEYWORDS: hexagonal boron nitride, chemical vapor deposition, electrical homogeneity, conductive AFM, polycrystalline. 


\section{ABSTRACT}

Hexagonal boron nitride ( $h$-BN) is a two dimensional (2D) layered insulator with superior dielectric performance that offers excellent interaction with other 2D materials (e.g. graphene, $\mathrm{MoS}_{2}$ ). Large-area $h$-BN can be readily grown on metallic substrates via chemical vapor deposition (CVD), but the impact of local inhomogeneities on the electrical properties of the $h$ $\mathrm{BN}$ and their effect in electronic devices is unknown. Here it is shown that the electrical properties of $h$-BN stacks grown on polycrystalline Pt vary a lot depending on the crystalline orientation of the Pt grain, but within the same grain the electrical properties of the $h$-BN are very homogeneous. The reason is that the thickness of the CVD-grown $h$-BN stack is different on each Pt grain. Conductive atomic force microscopy (CAFM) maps show that the tunneling current across the $h$-BN stack fluctuates up to 3 orders of magnitude from one Pt grain to another. However, probe station experiments reveal that the variability of electronic devices fabricated within the same Pt grain is surprisingly small. As cutting-edge electronic devices are ultra-scaled, and as the size of the metallic substrate grains can easily exceed $100 \mu \mathrm{m}$ (in diameter), CVDgrown $h$-BN stacks may be useful to fabricate electronic devices with low variability.

\section{MAIN TEXT}

The integration of two dimensional (2D) layered materials in electronic devices is a very successful strategy to enhance their intrinsic performance, as well as to provide additional functionalities such as flexibility and transparency. ${ }^{1}$ Conductive and semiconducting layered materials (e.g. graphene, $\mathrm{MoS}_{2}$ ) have been already integrated in many types of devices, including

field effect transistors (FETs), ${ }^{2}$ supercapacitors and sensors. ${ }^{3}$ In all these prototypes, the 2D 
layered materials need to interact with dielectrics (insulators) in order to provide electrical fields and capacitance effects. Unfortunately, the interaction between 2D materials (e.g. graphene, $\mathrm{MoS}_{2}$ ) and traditional dielectrics (e.g. $\mathrm{SiO}_{2}, \mathrm{HfO}_{2}, \mathrm{Al}_{2} \mathrm{O}_{3}$ ) is very problematic, due to defective bonding and high scattering at the interface. ${ }^{4}$ Hexagonal boron nitride $(h$-BN) is a layered insulator (direct band gap $\sim 5.9 \mathrm{eV}$ ), ${ }^{5}$ in which boron and nitrogen atoms arrange in a $\mathrm{sp}^{2}$ hexagonal structure by covalent bonding, whereas the layers stick to each other by van der Waals attraction. Given its high in-plane mechanical strength $(500 \mathrm{~N} / \mathrm{m}),{ }^{6}$ large thermal conductivity $\left(600 \mathrm{Wm}^{-1} \mathrm{~K}^{-1}\right),{ }^{7}$ and high chemical stability (up to $1500{ }^{\circ} \mathrm{C}$ in air), ${ }^{8} h$-BN has attracted much attention for a wide range of potential applications. For example, given its ultra-flat and surface free of dangling bonds $h$-BN substrates can increase the mobility of graphene-based FETs up to $\sim 140,000 \mathrm{~cm}^{2} \mathrm{~V}^{-1} \mathrm{~s}^{-19}$ (on $\mathrm{SiO}_{2}$ substrates it is lower, $15,000 \mathrm{~cm}^{2} \mathrm{~V}^{-1} \mathrm{~s}^{-1}$ ). When used as dielectric, $h$-BN has shown enhanced reliability (compared to $\mathrm{HfO}_{2}$ ), ${ }^{10}$ characteristic layer-by-layer dielectric breakdown process, ${ }^{11}$ and resistive switching. ${ }^{12}$

The first work on 2D $h$-BN (dating from 2005) isolated multilayer nanosheets (area $<100$ $\mu \mathrm{m}^{2}$ ) via mechanical exfoliation. ${ }^{13}$ As this technique is non-scalable, developing other methodologies for $h$-BN fabrication is of utmost importance. ${ }^{9}$ Liquid-phase exfoliation, molecular beam epitaxy and physical vapor deposition have been also suggested for $h$-BN preparation, ${ }^{11}$ but these approaches lead to abundant local defects and dangling bonds, which reduce the overall performance of the $h$-BN. Chemical vapor deposition (CVD) is an attractive approach to synthesize large-area $h$-BN stacks with low density of defects, in which the size of the samples is only limited by the size of furnace tube. Monolayer $h$-BN was firstly synthesized via CVD in ultra high vacuum (UHV) systems on single-crystal transition metals, such as $\mathrm{Pt}$ $(111)^{14}$ and Ni (111) ${ }^{15}$ but the complexity of the UHV system hindered its widespread. Later 
reports presented the CVD synthesis of monolayer sheets and few-layers-thick stacks of $h$-BN on metallic foils (e.g. $\mathrm{Cu}, \mathrm{Fe}, \mathrm{Pt})$ at atmospheric pressure (APCVD) and low pressure (LPCVD) ${ }^{16-19}$ It should be highlighted that, due to the high temperatures required for the CVD growth of $h$-BN $\left(>800^{\circ} \mathrm{C}\right)$, the metallic substrates become polycrystalline. Ref. ${ }^{19}$ reported that the thickness of the $h$-BN stack grown by CVD on polycrystalline $\mathrm{Ni}$ depends on the size of the Ni crystal underneath, and it was also suggested that $h$-BN grows faster on the surface of $\mathrm{Ni}(100)$ than $\mathrm{Ni}$ (111), due to the different catalytic reaction activities. ${ }^{20}$ Similar observations have been recently reported for $h$-BN stacks grown via CVD on polycrystalline Pt substrates. ${ }^{21}$ However, the impact of these thickness fluctuations on the local electrical properties of the $h$-BN stacks and their effect on the performance of electronic devices is still unknown. This information is essential to understand and control the device-to-device variability, which has been reported as one of the major problems of ultra-scaled technologies. ${ }^{22}$

In this work, the electrical homogeneity of continuous, large-area and high-quality $h$-BN stacks (grown by LPCVD on Pt substrates) is investigated via conductive atomic force microscopy (CAFM) and probe station. We find that thicker $h$-BN preferably grows on Pt grains with (101) crystallographic orientation. The excellent topographic-current correlation observed in CAFM maps indicates that the tunneling current across the $h$-BN is homogeneous within each Pt grain, but very different from grain to grain. Device level tests revealed that the variability of the devices fabricated within the same Pt grain is very small, and that the properties of devices grown on different Pt grains are strongly different to each other. The results here presented provide new insights on the electrical homogeneity of large-area $h$-BN stacks, and contribute to understand the variability of $h$-BN based electronic devices. 
Figure 1a shows the schematic of the LPCVD process for $h$-BN growth. A $1 \mathrm{~cm} \times 2.5 \mathrm{~cm}$ Pt substrate was cleaned via thermal annealing (see experimental section) and introduced in the center of the CVD tube (see Figure 1a). We use Pt as substrate because, despite being more expensive than $\mathrm{Cu}, \mathrm{Ni}$ or $\mathrm{Fe}$, the quality of the $h$-BN grown on $\mathrm{Pt}$ is higher (i.e. it holds a better layer structure with less randomly oriented crystallities). ${ }^{23}$ Liquid-phase borazine precursor was kept in a commercial cold container at $3{ }^{\circ} \mathrm{C}$ to avoid it's self-decomposition, and a cold trap filled with liquid nitrogen was used to prevent the damage of the pump. The borazine molecules (0.1 sccm) were delivered to the Pt substrate on $\mathrm{H}_{2}$ carrier gas $(70 \mathrm{sccm})$ at $950{ }^{\circ} \mathrm{C}$, which produced their absorption and decomposition on the surface of the Pt substrate, and the selfmediated growth of $h$-BN. After the LPCVD growth, the $h$-BN stack was transferred onto 300 $\mathrm{nm}-\mathrm{SiO}_{2} / \mathrm{Si}$ for Raman spectroscopy and optical microscopy inspection, and on metallic grids for transmission electron microscopy (TEM) characterization. The transfer of the $h$-BN was carried out following the bubbling approach based on water electrolysis (see Figure $1 \mathrm{~b}$ and the experimental section). ${ }^{23}$ This method is beneficial because it allows recycling the Pt foil for unlimited times (i.e. it is cost-effective).

Figure $2 \mathrm{a}$ and $2 \mathrm{~b}$ show the optical image and electron backscatter diffraction (EBSD) map of a Pt substrate after the thermal annealing (right before $h$-BN growth). Different Pt grains and crystallographic orientations can be distinguished. A long marker at the bottom right part of the image was made with a razor blade to identify this location in subsequent analyses. After that, multilayer $h$-BN was grown directly on the surface of the Pt substrate and transferred onto flat $300-\mathrm{nm}-\mathrm{SiO}_{2} / \mathrm{Si}$. The optical image of the $h-\mathrm{BN} / 300-\mathrm{nm}-\mathrm{SiO}_{2} / \mathrm{Si}$ (Figure $2 \mathrm{c}$ ) reveals regions with different colors (dark-green to light-green) perfectly matching the shapes of the Pt grains before $h$-BN growth (Figures $2 \mathrm{a}$ and $2 \mathrm{~b}$ ). Figure $2 \mathrm{c}$ also proves that the $h$-BN stack is continuous, as 
well as the non-destructive nature of the bubbling transfer method. Interestingly, the $h$-BN stacks grown on Pt grains with crystallographic orientations near (101) show light-green colors in the optical microscope image (see Figure 2c), which has been attributed to a larger thickness. ${ }^{19,21}$ This hypothesis has been verified by collecting Raman spectra at different locations on the surface of the $h-\mathrm{BN} / 300-\mathrm{nm}-\mathrm{SiO}_{2} / \mathrm{Si}$ sample (see Figure $2 \mathrm{~d}$ ). The dark-green grains in the optical microscope image (Figure 2c) always showed an $E_{2 g}$ peak near $1370 \mathrm{~cm}^{-1}$, which corresponds to monolayer $h$-BN; on the contrary at the light-green grains the $\mathrm{E}_{2 \mathrm{~g}}$ peak shifted towards $\sim 1366$ $\mathrm{cm}^{-1}$, which is the characteristic value of bulk $h$-BN. High magnification TEM images (Figure 2e) present the definitive corroboration of grain-dependent thickness in the $h$-BN stack. The thicknesses observed via TEM at multiple locations of the sample (Figure 2e) always ranged between 1-2 and 10-13 layers, meaning that the dark and light regions in Figure 2c should correspond to thicknesses of 1-2 and 10-13 layers, respectively. It is worth noting that, for all thicknesses, the TEM images reveal defect-free layered structure. The electron energy loss spectroscopy (EELS) spectrum (Figure 2f) shows two edges at around 180 and $390 \mathrm{eV}$, which correspond to the characteristic k-shell ionization edges of boron and nitrogen. This verifies that the stoichiometric ratio of boron and nitrogen is 1:1, which is characteristic of layered $h$-BN with hexagonal lattice. ${ }^{6}$

The surface roughness of the $h$-BN grown on Pt grains with different orientations has been analyzed via atomic force microscopy (AFM). Figure S1 shows the topographic AFM maps measured on the surface of the $300-\mathrm{nm}-\mathrm{SiO}_{2} / \mathrm{Si}$ sample shown in Figure 2c; the root mean square (RMS) roughness of each image (calculated via AFM software) is also displayed. As it can be observed, the flattest surface is detected for the $h$-BN that was grown on Pt (111), and the roughest corresponds to the $h-\mathrm{BN}$ that was grown on Pt (101). As the surface roughness of 2D 
materials increase with their thickness, ${ }^{24}$ Figure $\mathrm{S} 1$ further supports that the thinnest $h$-BN stack grows on Pt (111), and that the thickest grows on Pt (101). Moreover, multilayer islands have been detected on the surface of the $h$-BN grown on Pt (001) and Pt (101), as shown in Figure S2, further suggesting the growth of thicker $h$-BN stacks. Alternative methods to evaluate the thickness of the h-BN stack are low energy electron microscopy (LEEM) and low energy electron diffraction (LEED), ${ }^{25}$ which may be used in future works.

The electrical performance of as-grown $h-\mathrm{BN} / \mathrm{Pt}$ (without transfer) has been tested via CAFM. Figures $3 \mathrm{a}$ and $3 \mathrm{~b}$ show the simultaneously collected topographic and current maps (respectively) measured on the surface of the $h$-BN stack when applying a bias of $-2 \mathrm{~V}$ to the $\mathrm{Pt}$ substrate (CAFM tip grounded). In order to analyze the electrical properties of $h$-BN grown on different Pt grains, we used a large $(80 \mu \mathrm{m} \times 80 \mu \mathrm{m})$ scan size and strategically selected an area of the sample containing several Pt grains. The different Pt grains detected have been named with letters from A to G. Despite the $h$-BN cannot be detected in the topographic map (it is very flat compared to the Pt surface, see Figure S1), its effect can be clearly seen in the current map (Figure 3b), which reveals sharp conductivity changes from grain to grain. The different currents collected on each Pt grain must be related to the presence of $h$-BN stacks with different thicknesses because the underlying Pt grains, despite having different crystal orientations, hold similar conductivities. ${ }^{26}$ It should be highlighted that the $h$-BN adhesion to the Pt surface is always by van der Waals forces for any Pt crystalline orientation, which means that the electronic coupling between the h-BN and Pt from one grain to another does not change. Therefore, h-BN/Pt electronic coupling is not one factor producing the conductivity changes from one grain to another observed in Figure $3 b$. The fact that the conductivity changes have been detected in the same image discards tip wearing from one grain to the other, and the perfect 
topography-current correlation undoubtedly demonstrate that $h$-BN grown on different grains hold different conductivities (due to their different thicknesses, as shown in Figure 2). It should be highlighted that, sporadically, the regions close to the Pt grain boundaries have shown higher currents (see for example the grain boundaries between grains D-G and F-G). The explanation for this observation is as follows: when the metallic substrate is exposed to large temperatures it becomes polycrystalline; the grain boundaries of the metal substrate are rough and may contain asperities; these topographic accidents alter the h-BN growth, and at that location the h-BN may be thinner or even cracked, displaying large currents in the current maps. Cross-sections have been collected (offline) at all the grains of the current image (Figure 3b) using the AFM software (NanoScope Analysis) and assembled one after the other (using OriginPro 8 software). The result is displayed in Figure 3c. Within each grain the current is homogeneous, and sharp changes are detected from grain to grain. As it can be observed, the highest currents are detected on grain B, indicating that it holds the thinnest $h$-BN stack on its surface. Grain $\mathrm{C}$ shows negligible currents similar to the electrical noise of the CAFM, indicating that the $-2 \mathrm{~V}$ applied were not enough to generate tunneling current across the $h$-BN stack. It should be emphasized that the current deviations within each grain are below one order of magnitude for all grains (compare maximum and minimum peaks within each grain in Figure 3c). This value is smaller than that observed in other thin insulating films (of similar thickness) being currently used in the industry, such as $\mathrm{HfO}_{2}$ and $\mathrm{TiO}_{2}$ (see Figure S3). Therefore, the electrical properties of h-BN within the same grain seem to be very homogeneous, which shows great potential to mitigate device-to-device variability problems of ultra scaled devices. Further electrical information about the grains has been obtained by measuring the onset voltage $\left(\mathrm{V}_{\mathrm{ON}}\right)$ of the $h$-BN stacks on each Pt grain. The onset voltage is defined as the minimum voltage that needs to be applied between the CAFM tip 
and the substrate of the sample (Pt) in order to observe tunneling currents above the noise level. ${ }^{27}$ Despite the noise level of our CAFM is $\sim 1 \mathrm{pA}$, we selected $\mathrm{V}_{\mathrm{ON}}=\mathrm{V}(\mathrm{I}=5 \mathrm{pA})$ in order to be completely sure that non-negligible current is flowing across the $h$-BN stack. For this experiment, $V_{\text {ON }}$ has been extracted by measuring individual current maps on each grain $(1 \mu \mathrm{m} \times 1 \mu \mathrm{m})$. The results obtained (Table S1), strongly support the observations in Figure 3: the smallest VON (0.1 V) was detected on grain $\mathrm{B}$, and the highest $(6 \mathrm{~V})$ on grain $\mathrm{C}$.

In Figure $3 \mathrm{c}$ it is difficult to quantify the real thickness of the h-BN on each Pt grain, and correlate it with the current levels observed. The reason is that best techniques used for physical thickness characterization of 2D materials (i.e. TEM) are destructive. In order to provide more insights to this point here we perform an additional analysis, consisting on measuring I-V curves at different locations of each grain via CAFM. Based on the shape of the I-V curves showing the tunneling current across the insulating stack, its physical thickness can be calculated very accurately (this was done before for ultra thin $\mathrm{SiO}_{2}$ films with sub-nanometer resolution. ${ }^{28}$ In previous works ${ }^{29}$ it was determined that the dominant conduction across multilayer h-BN stacks was by Forler-Nordheim Tunneling (FNT). Therefore, here we use the FNT equation to calculate the tunneling current for different h-BN thicknesses:

$$
I=\frac{A_{e f f} \sqrt{m \phi_{B}} q^{2} V}{h^{2} d} \exp \left[\frac{-4 \pi \sqrt{m \phi_{B}} d}{h}\right]
$$

where $V$ is the applied voltage, $d$ is the thickness of the h-BN, $A_{\text {eff }}$ is the effective contact area, $\phi_{B}$ is the barrier height. The parameters $m, q$ and $h$ are the free electron mass, the electron charge and the Planck constant, respectively. This calculation has been repeated for different values of $d$ depending on the number of layers $(\mathrm{N})$, that is $\mathrm{d}=0.33 \mathrm{~nm}$ for $\mathrm{N}=1, \mathrm{~d}=0.66 \mathrm{~nm}$ for $\mathrm{N}=2$, etc... 
having increments of $0.33 \mathrm{~nm}$ for each layer until $\mathrm{N}=24$. By comparing the calculations with the I-V curves experimentally collected (see Figure S4), it can be concluded that the tunneling currents across grain $\mathrm{B}$ fits well the conduction of monolayer h-BN, while the tunneling currents across grain D fit well the conduction across 11-13 layers (see Figure S4). This result is interesting because provides an indirect quantification of the thickness at each grain, something that the CAFM maps (Figure 3b) cannot provide.

Finally, the electrical properties of $h$-BN stacks grown on polycrystalline Pt substrates have been analyzed at the device level. To do so, squared $\mathrm{Au} / \mathrm{Ti}$ top electrodes with lateral sizes ranging from $10 \mu \mathrm{m} \times 10 \mu \mathrm{m}$ to $100 \mu \mathrm{m} \times 100 \mu \mathrm{m}$ have been evaporated on the surface of the $h$ $\mathrm{BN} / \mathrm{Pt}$ samples (see experimental section), leading to matrixes of metal/insulator/metal (MIM) cells (i.e. Au/Ti/h-BN/Pt). MIM cells are used in several devices, including FETs, ${ }^{2}$ capacitors, ${ }^{3}$ and memristors,${ }^{30}$ as well as in test structures to evaluate essential parameters of the insulator, such as charge trapping, stress induced leakage current, dielectric breakdown (BD) and resistive switching (RS). ${ }^{31}$ Therefore, despite holding an easy structure, the MIM cells fabricated are representative of $h$-BN based microelectronic devices. Figure 4a shows the schematic of asgrown $h$-BN on polycrystalline Pt foil before and after electrodes deposition, in which $h$-BN film serves as insulating layer between the top $(\mathrm{Au} / \mathrm{Ti})$ and bottom $(\mathrm{Pt})$ metal electrodes. The optical microscope image of the sample after Au/Ti electrode deposition is shown in Figure $4 \mathrm{~b}$. The corresponding EBSD map of the same area of the sample has been also collected (Figure 4c). It should be highlighted that, unlike in Figure 2b, in Figure 4c the EBSD map has been collected in the presence of $h$ - $\mathrm{BN}$ on the $\mathrm{Pt}$ substrate. As the $h$ - $\mathrm{BN}$ is an insulator, this may distort the signal related to the local crystallographic orientation. For this reason, the EBSD map in Figure 4d will be only used to distinguish different grains (in that case the contrast is large) but not to identify 
which is the real crystallographic orientation of the Pt within each grain. For this reason, the color scale in Figure $4 \mathrm{c}$ has been intentionally removed. Several devices on the same and different Pt grains have been tested in the probe station by applying ramped voltage stresses (RVS), and the resulting current vs. voltage (I-V) curves have been compared. As an example, Figure $4 \mathrm{~d}$ shows the I-V curves collected on two devices with the same size $(25 \mu \mathrm{m} \times 25 \mu \mathrm{m})$ that belong to the same Pt grain (devices 1 and 2 in Figure 4c). As it can be observed, the I-V characteristics for devices 1 and 2 are strikingly similar: $i$ ) the $\mathrm{BD}$ voltages $\left(\mathrm{V}_{\mathrm{BD}}\right)$ show very small deviation $(0.55 \mathrm{~V}$ and $0.61 \mathrm{~V})$; ii) the pre- and post-BD currents (IPRE-BD and IPOST-BD, respectively) almost overlap, and iii) the IPOST-BD/IPRE-BD ratio is identical. These results are indeed indicating that the device-to-device variability within the same Pt grain is very small. Any potential variability of the pre-breakdown I-V curves in devices within the same Pt grain should be related to nanoscale inhomogeneities within the h-BN stack, such as thickness fluctuations (see Figure S2), local defects, h-BN domain boundaries and/or wrinkles. Nevertheless, as displayed in Figures $4 c, 3 c$ and $S 3$ the variability of the electrical properties of the h-BN within the same Pt grain are very small. On the contrary, the devices with the same size but patterned on different Pt grains show very inhomogeneous I-V characteristics. As an example, Figure 4e shows the I-V curves collected on devices 3 and 4 (see Figure 4c). First, the pre-BD currents are very different; second, $\mathrm{V}_{\mathrm{BD}}$ for both devices are remarkably different: $2.09 \mathrm{~V}$ for device 3 and $0.89 \mathrm{~V}$ for device 4; and third, the IPOST-BD/IPRE-BD ratio is also slightly different. The different electrical properties of devices 3 and 4 are related to the different thicknesses of the $h$-BN stack, due to the different crystallographic orientation of the underlying Pt grain. These observations have been corroborated by measuring additional MIM devices at different Pt grains. As Figure $4 \mathrm{f}$ shows, the deviation of $\mathrm{V}_{\mathrm{BD}}$ within each grain is very small (from $\pm 0.013 \mathrm{~V}$ for grain $\mathrm{C}$ to 
$\pm 0.054 \mathrm{~V}$ for grain $\mathrm{B}$ ), but the deviations of $\mathrm{V}_{\mathrm{BD}}$ from one Pt grain to another are large (from $0.58 \mathrm{~V}$ in grain $\mathrm{A}$ to $2.13 \mathrm{~V}$ in grain $\mathrm{D}$ ). The breakdown event observed for all devices (displayed as a sharp current increase in Figures $4 \mathrm{~d}$ and $4 \mathrm{e}$ ) further demonstrates that the $h$-BN sheet grown on the Pt substrate is continuous, otherwise a larger current under smaller voltage (i.e. 10-2 $\mathrm{A} @ 0.1 \mathrm{~V}$ ) typical of shorted devices should be observed.

Fortunately, cutting-edge electronic devices based on MIM cells cover ultra scaled areas. In the case of FETs, the current technology node is $7 \mathrm{~nm}$, and the total length of current FETs never exceeds $50 \mathrm{~nm}$. In the case of memristors and other non-volatile memories, such as resistive random access memories (RRAM), phase change random access memories (PCRAM) and ferroelectric random access memories (FeRAM), device areas down to $10 \mathrm{~nm} \times 10 \mathrm{~nm}$ are preferred. ${ }^{32}$ Therefore, as the diameter of the Pt grains easily surpass $100 \mu \mathrm{m}$, the fabrication of $h$-BN based electronic devices with very low variability is feasible. More efforts towards the growth of large-area single-crystalline $h$-BN stacks should conduct to ultra-low variability technologies.

In conclusion, the electrical homogeneity of $h$-BN stacks grown via CVD on Pt substrates has been analyzed by CAFM and a probe station. We observe that $h$-BN grows thicker on $\mathrm{Pt}$ grains with crystallographic orientations close to (101). In situ CAFM characterization reveals that the tunneling current across the $h$-BN grown on the same Pt grain is very homogeneous (i.e. more homogeneous than that observed in other insulators being currently used in the industry, such as $\mathrm{HfO}_{2}$ and $\mathrm{TiO}_{2}$ ), but sharp conductivity changes are detected from grain to grain. Device level tests in the probestation reveal that the variability of $\mathrm{Au} / \mathrm{Ti} / h$-BN/Pt devices within each grain is strikingly low in terms of IPRE-BD, IPOST-BD and $V_{B D}$. These results contribute to the 
understanding of the electrical properties of $h$-BN and variability of $h$-BN based electronic devices.

\section{Experimental Section}

Growth of h-BN Pt substrates: High purity (99.997\%) $100 \mu \mathrm{m}$ thick Pt foil purchased from Alfa Aesar (item no. 12059) was employed as substrate for the $h$-BN growth. First, the asreceived Pt foil was cleaned in acetone and isopropanol (IPA) for 10 minutes to remove the surface contamination, and dried with a nitrogen gun. Then, the Pt foil was introduced in the center of the quartz tube, and the temperature ramped up to $950{ }^{\circ} \mathrm{C}$ in $70 \mathrm{sccm} \mathrm{H}_{2}$ atmosphere. The time required to increase the temperature from $\sim 20{ }^{\circ} \mathrm{C}$ to $950{ }^{\circ} \mathrm{C}$ was $\sim 40$ minutes, and following by the annealing process under the temperature of $950{ }^{\circ} \mathrm{C}$ in $70 \mathrm{sccm} \mathrm{H}_{2}$ for 30 minutes, in order to remove the contamination contains carbon or oxygen. This pre-growth heating step is called annealing, and it is useful to clean impurities on the Pt surface. Then, the valve of the tube coming from the Borazine container (which used a $\mathrm{H}_{2}$ flow rate of $0.1 \mathrm{sccm}$ ) was opened, allowing the introduction of borazine molecules in the quarz tube containing the $\mathrm{Pt}$ substrate. This process was kept for 1 hour at $950{ }^{\circ} \mathrm{C}$. After that time, the temperature controller was set at room temperature and the CVD system was cooled down.

Transfer process for the $h$-BN: After sample fabrication, the $h$-BN stacks were transferred on $\mathrm{SiO}_{2} / \mathrm{Si}$ wafers and TEM grids for analysis. To do so, liquid poly(methyl methacrylate) (PMMA) from MicroChem was spin-coated on both sides of the $h$-BN/Pt $/ h$-BN sample at 2500 $\mathrm{rpm}$ for $1 \mathrm{~min}$. The sample was backed in the oven at $70{ }^{\circ} \mathrm{C}$ for $10 \mathrm{~min}$ to solidify the PMMA, and the resulting sample (PMMA $/ h$-BN/Pt/h-BN/PMMA) was immersed in a container filled with $1 \mathrm{M} \mathrm{NaOH}$. In the same container, a Pt mesh was also introduced, and a potential difference 
of $3 \mathrm{~V}$ between it and the PMMA/h-BN/Pt/h-BN/PMMA sample was applied using a source meter. The Pt mesh served as anode, and the PMMA/h-BN/Pt/h-BN/PMMA sample as cathode. The application of voltage lead to the formation of hydrogen bubbles at the $h$-BN/Pt interface, leading to the effective PMMA/h-BN detachment from the Pt foil ${ }^{33}$ in less than 10 minutes. Afterwards, the PMMA/ $h$-BN stack was cleaned in deionized water three times to remove the residual $\mathrm{NaOH}$ solution. Finally, $\mathrm{PMMA} / h$-BN was transferred on the target substrates $\left(\mathrm{SiO}_{2} / \mathrm{Si}\right.$ or TEM grids). When transferred on the $\mathrm{SiO}_{2} / \mathrm{Si}$ substrate the PMMA was removed by soaking the entire sample in acetone for 2 hours, followed by an annealing at $400{ }^{\circ} \mathrm{C}$ for $2 \mathrm{~h}$ in a mixed $\mathrm{H}_{2}(200 \mathrm{sccm})$ and $\mathrm{Ar}(200 \mathrm{sccm})$ atmosphere. When transferred on the TEM grids, only the annealing step was used (no sample soaking because that could damage the $h$-BN suspended on the perforated TEM grid).

$h-B N$ and $P t$ characterization: The different Pt grains and their crystallographic orientations were analyzed using a standard optical microscope, and an EBSD system (from Oxford Technology) integrated in a scanning electron microscope (Zeiss Merlin HRSEM). The topographic maps in Figures S1-S2 were collected using a Dimension 3000 AFM working in air atmosphere. These experiments were performed in tapping mode using $\mathrm{Si}$ probe tips from Budgetsensors (model: Tap300-G). The CAFM experiments were carried out in a Multimode VI equipment from Veeco working inside a nitrogen chamber (relative humidity $\sim 0.5 \%$ ). ${ }^{34}$ The use of a nitrogen atmosphere is beneficial to stabilize the current signal and achieve larger lateral resolution. ${ }^{35}$ For this experiment we used PtIr varnished Si probes from Nanosensors (model: CONTPT). The cross section in the current CAFM maps have been calculated using the NanoScope Analysis software of the AFM (Bruker) and assempbled using OriginPro 8 software. Atomic scale information about the thickness and quality of the $h$-BN stacks was collected using 
a JEOL 2010 HRTEM with EELS capability integrated, and a LabRAM Raman spectrometer from Horiba.

Device fabrication and characterization: Squared metallic top electrodes with lateral sizes ranging from $10 \mu \mathrm{m} \times 10 \mu \mathrm{m}$ to $100 \mu \mathrm{m} \times 100 \mu \mathrm{m}$ have been deposited on as-grown $h$-BN/Pt samples. First $20 \mathrm{~nm} \mathrm{Ti}$ and second $60 \mathrm{~nm}$ Au have been deposited via E-beam evaporator (Ajaint AJA-ATC) using a laser-patterned shadow mask. The electrical measurements were carried out in a Summit 11000 AP probe station connected to an Agilent 4155C semiconductor parameter analyzer. The RVS was applied to the top electrodes and the Pt substrates were grounded.

\section{Supporting Information.}

The Supporting Information are available free of charge.

Additional AFM characterization of the $h-\mathrm{BN} / 300-\mathrm{nm}-\mathrm{SiO}_{2} / \mathrm{Si}$ sample and Electrical measurements are conducted on each grain will be found in Supporting Information. (PDF)

\section{AUTHOR INFORMATION}

\section{Corresponding Author}

Mario Lanza, email: mlanza@suda.edu.cn

\section{Author Contributions}

The manuscript was written through contributions of all authors. All authors have given approval to the final version of the manuscript. $\$$ These authors contributed equally. 


\section{ACKNOWLEDGMENT}

Fei Hui and Wenjing Fang contributed equally to this work. F. Hui acknowledges the support from the Young 1000 Global Talent Recruitment Program of the Ministry of Education of China, the National Natural Science Foundation of China (grants no. 61502326, 41550110223, 11661131002), the Jiangsu Government (grant no. BK20150343), the Ministry of Finance of China (grant no. SX21400213) and the Young 973 National Program of the Chinese Ministry of Science and Technology (grant no. 2015CB932700). The Collaborative Innovation Center of Suzhou Nano Science \& Technology, the Jiangsu Key Laboratory for Carbon-Based Functional Materials \& Devices, the Priority Academic Program Development of Jiangsu Higher Education Institutions, and the Opening Project of Key Laboratory of Microelectronic Devices \& Integrated Technology (Institute of Microelectronics, Chinese Academy of Sciences) are also acknowledged. W. Fang, T. Kpulun, G. Harris and J. Kong acknowledge the support from the STC Center for Integrated Quantum Materials, NSF Grant No. DMR-1231319. H. Wang and J. Kong acknowledge the support from NSF DMR/ECCS - 1509197. W.S. Leong acknowledges the support from SUTD-MIT Postdoctoral Fellows Program.

\section{REFERENCES}

(1) Choi, D.; Choi, M Y.; Choi, W. M.; Shin, H. J.; Park, H. K.; Seo, J. S.; Park, J.; Yoon, S. M.; Chae, S. J.; Lee, Y. H.; Kim, S. W.; Choi, J. K.; Lee, S. Y.; Kim, J. M. Fully Rollable Transparent Nanogenerators Based on Graphene Electrodes. Adv. Mater. 2010, 22, $2187-$ 2192. 
(2) Kim, B. J.; Lee, S. K.; Kang, M. S.; Ahn, J. Y.; Cho, J. H. Coplanar-Gate Transparent Graphene Transistors and Inverters on Plastic. ACS Nano, 2012, 6, 8646-8651.

(3) Guo, N.; Wei, J.; Jia, Y.; Sun, H.; Wang, Y.; Zhao, K.; Shi, X.; Zhang, L.; Li, X.; Cao, A. Fabrication of Large Area Hexagonal Boron Nitride Thin Films for Bendable Capacitors. Nano Res. 2013, 6, 602-610.

(4) McDonnell, S.; Brennan, B.; Azcatl, A.; Lu, N.; Dong, H.; Buie, C.; Kim, J.; Hinkle, C. L.; Kim, M. J.; Wallace, R. M. $\mathrm{HfO}_{2}$ on $\mathrm{MoS}_{2}$ by Atomic Layer Deposition: Adsorption Mechanisms and Thickness Scalability. ACS Nano 2013, 7, 10354-10361.

(5) Watanabe, K.; Taniguchi, T.; Kanda, H. Direct-bandgap Properties and Evidence for Ultraviolet Lasing of Hexagonal Boron Nitride Single Crystal. Nat. Mater. 2004, 3, 404409.

(6) Song, L.; Ci, L. J.; Lu, H.; Sorokin, P. B.; Jin, C. H.; Ni, J.; Kvashnin, A. G.; Kvashnin, D. G.; Lou, J.; Yakobson, B. I.; Ajayan, P. M. Large Scale Growth and Characterization of Atomic Hexagonal Boron Nitride Layers. Nano Lett. 2010, 10, 3209-3215.

(7) Lindsay, L.; Broido, D. A. Enhanced Thermal Conductivity and Isotope Effect in SingleLayer Hexagonal Boron Nitride. Phys. Rev, B 2011, 84, 155421.

(8) Liu, Z.; Gong, Y. J.; Zhou, W.; Ma, L. L.; Yu, J. J.; Idrobo, J. C.; Jung, J.; MacDonald, A. H.; Vajtai, R.; Lou, J.; Ajayan, P. M. Ultrathin High-Temperature Oxidation-Resistant Coatings of Hexagonal Boron Nitride. Nat. Commun. 2013, 4, 2541.

(9) Dean, C. R.; Young, A. F.; Meric, I.; Lee, C.; Wang, L.; Sorgenfrei, S.; Watanabe, K.; Taniguchi, T.; Kim, P.; Shepard, K. L.; Hone, J. Boron Nitride Substrates for HighQuality Graphene Electronics. Nat. Nanotechnol. 2010, 5, 722-726. 
(10) Ji, Y. F.; Pan, C. B.; Zhang, M. Y.; Long, S. B.; Lian, X. J.; Miao, F.; Hui, F.; Shi, Y. Y.; Larcher, L.; Wu, E.; Lanza, M. Boron Nitride as Two Dimensional Dielectric: Reliability and Dielectric Breakdown. Appl. Phys. Lett. 2016, 108, 012905.

(11) Hui, F.; Pan, C. B.; Shi, Y. Y.; Ji, Y. F.; Gutierrez, E. G.; Lanza, M. On the Use of Two Dimensional Hexagonal Boron Nitride as Dielectric. Microelecron. Eng. 2016, 163, 119133.

(12) Pan, C. B.; Ji, Y. F.; Xiao, N.; Hui, F.; Tang, K. C.; Guo, Y. Z.; Xie, X. M.; Puglisi, F. M.; Larcher, L.; Miranda, E.; Jiang, L. L.; Shi, Y. Y.; Valov, I.; McIntyre, P. C.; Waser, R.; Lanza, M. Coexistence of Grain-Boundaries-Assisted Bipolar and Threshold Resistive Switching in Multilayer Hexagonal Boron Nitride. Adv. Func. Mater. 2017, 1604811.

(13) Novoselov, K. S.; Jiang, D.; Schedin, F.; Booth, T. J.; Khotkevich, V. V.; Morozov, S. V.; Geim, A. K. Two-Dimensional Atomic Crystals. Porc. Natl. Acad. Sci. 2005, 102, 10451-10453.

(14) Nagashima, A.; Tejima, N.; Gamou, Y.; Kawai, T.; Oshima, C. Electronic States of Monolayer Hexagonal Boron Nitride Formed on the Metal Surfaces. Surf. Sci. 1996, 357, $307-311$.

(15) Nagashima, A.; Tejima, N.; Gamou, Y.; Kawai, T.; Oshima, C. Electronic Dispersion Relations of Monolayer Hexagonal Boron Nitride Formed on the Ni(111) Surface. Phys. Rev. B 1995, 51, 4606-4613.

(16) Kim, S. M.; Hsu, A.; Park, M. H.; Chae, S. H.; Yun, S. J.; Lee, J. S.; Cho, D. H.; Fang, W. J.; Lee, C. G.; Palacios, T.; Dresselhaus, M.; Kim, K. K.; Lee, Y. H.; Kong, J. Synthesis of Large-Area Multilayer Hexagonal Boron Nitride for High Material Performance. Nat. Commun. 2015, 6, 8662. 
(17) K. K. Kim, A. Hsu, X. T. Jia, S. M. Kim, Y. M. Shi, M. Hofmann, D. Nezich, J. F. Rodriguez-Nieva, M. Dresselhaus, T. Palacios and J. Kong, Nano Lett. 2012, 12, 161-166.

(18) Y. Gao, W. C. Ren, T. Ma, Z. B. Liu, Y. Zhang, W. B. Liu, L. P. Ma, X. L. Ma and H. M. Cheng, ACS Nano. 2013, 7, 5199.

(19) Shi, Y. M.; Hamsen, C.; Jia, X. T.; Kim, K. K.; Reina, A.; Hofmann, M.; Hsu, A. L.; Zhang, K.; Li, H.; Juang, Z. Y.; Dresselhaus, M. S.; Li, L. J.; Kong, J. Synthesis of FewLayer Hexagonal Boron Nitride Thin Film by Chemical Vapor Deposition. Nano Lett. 2010, $10,4134-4139$.

(20) Lee, Y. H.; Liu, K. K.; Lu, A. Y.; Wu, C. Y.; Lin, C. T.; Zhang, W. J.; Su, C. Y.; Hsu, C. L.; Lin, T. W.; Wei, K. H.; Shi, Y. M.; Li, L. J. Growth Selectivity of Hexagonal-Boron Nitride Layers on Ni with Various Crystal Orientations. RSC Adv. 2012, 2, 111-115.

(21) Park, J. H.; Park, J. C.; Yun, S. J.; Kim, H.; Luong, D. H.; Kim, S. M.; Choi, S. H.; Yang, W.; Kong, J.; Kim, K. K.; Lee, Y. H. Large-Area Monolayer Hexagonal Boron Nitride on Pt Foil. ACS Nano 2014, 8, 8520-8528.

(22) Allan, A. International Technology Roadmap for Semiconductors of 2008, https://cseweb.ucsd.edu/classes/wi09/cse242a/itrs/ORTC.pdf, accessed: February, 2016.

(23) Kim, G.; Jang, A. R.; Jeong, H. Y.; Lee, Z.; Kang, D. J.; Shin, H. S. Growth of HighCrystalline, Single-Layer Hexagonal Boron Nitride on Recyclable Platinum Foil. Nano Lett. 2013, 13, 1834-1839.

(24) Fang, W. J. Synthesis of Bilayer Graphene and Hexagonal Boron Nitride by Chemical Vapor Deposition Method. Ph.D. Thesis, Massachusetts of Institute Technology, Cambridge, US, 2015. 
(25) Ismach, A.; Chou, H.; Mende, P.; Dolocan, A.; Addou, R.; Aloni, S.; Wallace, R.; Feenstra, R.; Ruoff, R.S.; Colombo, L. Carbon-assisted chemical vapor deposition of hexagonal boron nitride. 2D Mater. 2017, 4, 025117.

(26) Powell, R. W.; Tye, R. P.; Woodman, M. J. The Thermal Conductivity and Electrical Resistivity of Polycrystalline Metals of the Platinum Group and of Single Crystals of Ruthenium. J. Less-Common Metals, 1967, 12, 1-10.

(27) Lanza, M.; Porti, M.; Nafría, M.; Aymerich, X.; Ghidini, G.; Sebastiani, A. Trapped Charge and Stress Induced Leakage Current (SILC) in Tunnel SiO2 Layers of Deprocessed MOS Non-Volatile Memory Devices Observed at the Nanoscale. Microelectron. Reliab. 2009, 49, 1188-1191.

(28) Frammelsberger, W.; Benstetter, G.; Kiely, J.; Stamp, R. CAFM-based thickness determination of thin and ultra-thin $\mathrm{SiO} 2$ films by use of different conductive-coated probe tips. Appl. Surf. Sci. 2007, 253, 3615-3626.

(29) Lee, G.H.; Yu, Y.J.; Lee, C.; Dean, C.; Shepard, K.L.; Kim, P.; Hone, J. Electron tunneling through atomically flat and ultrathin hexagonal nitride. Appl. Phys. Lett. 2011, $99,243114$.

(30) Puglisi, F. M.; Larcher, L.; Pan, C.; Xiao, N.; Shi, Y.; Hui, F.; Lanza, M. 2D h-BN Based RRAM Devices. IEEE Int. Electron Dev. Meeting 2016, 16, 874-877.

(31) Miranda, E.; Suñé, J.; Rodríguez, R.; Nafría M.; Aymerich, X. Soft Breakdown Fluctuation Events in Ultrathin $\mathrm{SiO}_{2}$ Layers. Appl. Phys. Lett. 1998, 73, 490-492.

(32) Govoreanu, B.; Kar, G. S.; Chen, Y. Y.; Paraschiv, V.; Fantini, A.; Radu, I. P.; Goux, L.; Clima, S.; Degraeve, R.; Jossart, N. 10×10nm2 Hf/HfOx Crossbar Resistive RAM with 
Excellent Performance, Reliability and Low-Energy Operation. IEEE Int. Electron Dev. Meeting, 2011, 11, 729-732.

(33) Gao, L. B.; Ren, W. C.; Xu, H. L.; Jin, L.; Wang, Z.; Ma, T.; Ma, L. P.; Zhang, Z. Y.; Fu, Q.; Peng, L. M.; Bao, X. H.; Cheng, H. M. Repeated Growth and Bubbling Transfer of Graphene with Millimeter-Size Single-Crystal Grains Using Platinum. Nat. Commun. 2012, 2, 699-705.

(34) Ji, Y. F.; Hui, F.; Shi, Y. Y.; Han, T. T.; Song, X. X.; Pan, C. B.; Lanza, M. Fabrication of a Fast-Response and User-Friendly Environmental Chamber for Atomic Force Microscopes. Rev. Sci. Inst. 2015, 86, 106105.

(35) Lanza, M.; Porti, M.; Nafría, M.; Aymerich, X.; Whittaker, E.; Hamilton, B. Electrical Resolution During Conductive Atomic Force Microscopy Measurements under Different Environmental Conditions and Contact Forces. Rev. Sci. Inst. 2010, 81, 106110. 


\section{FIGURES}

a

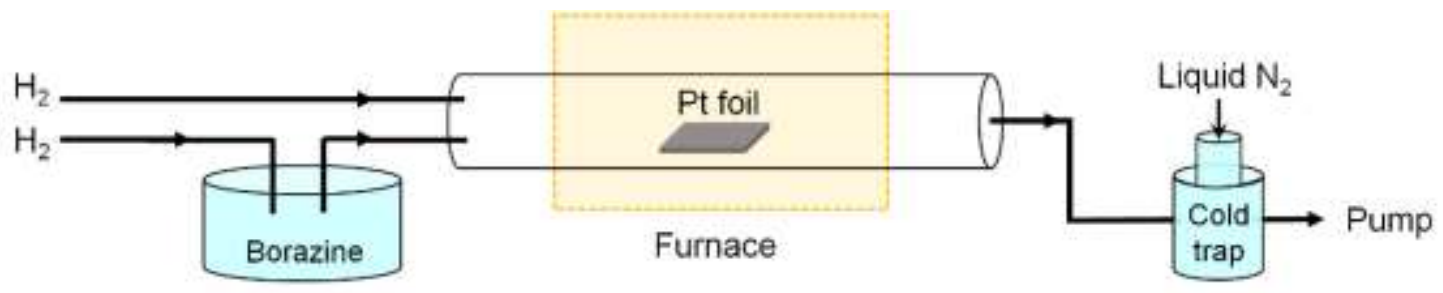

b

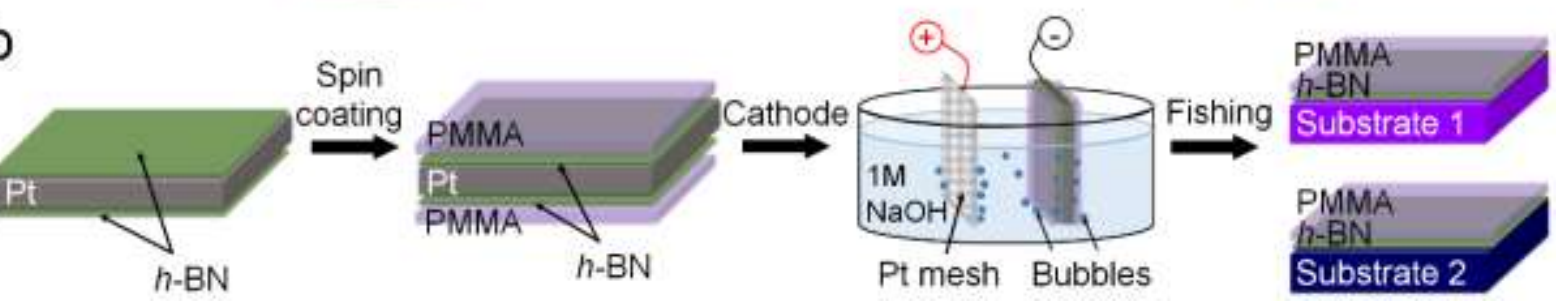

Figure 1. (a) Schematic of LPCVD process to grow $h$-BN on Pt foils. (b) Electrochemical (bubble) method to transfer $h$-BN from Pt foil to the target substrates. 

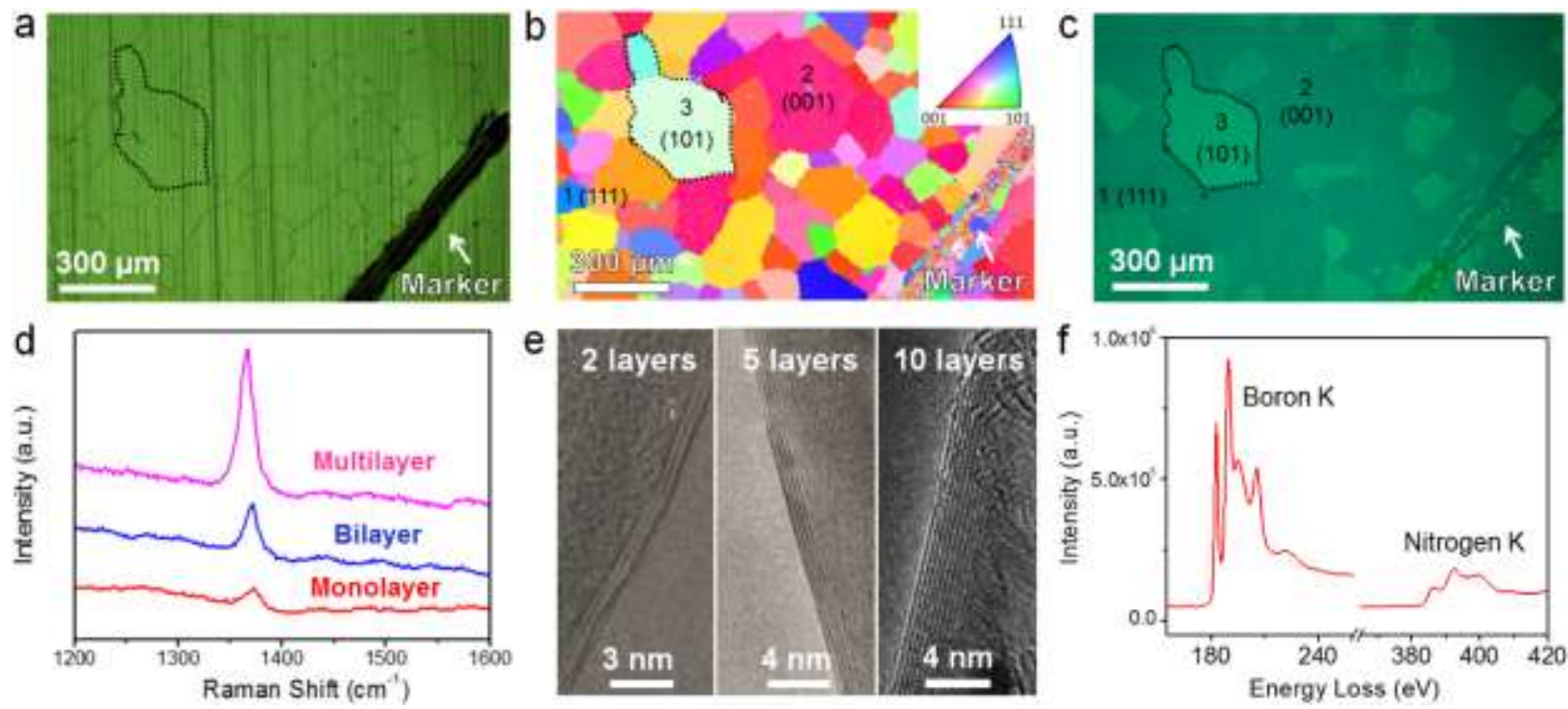

Figure 2. Characterization of $h$-BN films. (a) Optical microscope and (b) EBSD images of a polycrystalline Pt substrate before $h$-BN growth (annealed). The two images correspond to the same location of the sample, as highlighted by the marker in the right-bottom part. (c) Optical microscope image of a $h$ - $\mathrm{BN} / 300-\mathrm{nm}-\mathrm{SiO}_{2} / \mathrm{Si}$ sample. The $h-\mathrm{BN}$ was grown on the area displayed in (a). The granular pattern can be distinguished with different dark-green and lightgreen colors. (d) Raman spectra collected on different locations of the $h-\mathrm{BN} / 300-\mathrm{nm}-\mathrm{SiO}_{2} / \mathrm{Si}$ sample. Monolayer/multilayer have been collected at dark-green/light-green locations of panel (c). High resolution TEM images demonstrating the different thicknesses and the good layered structure of the $h$-BN stacks. (f) EELS spectrum of $h$-BN showing the typical boron and nitrogen peaks. 


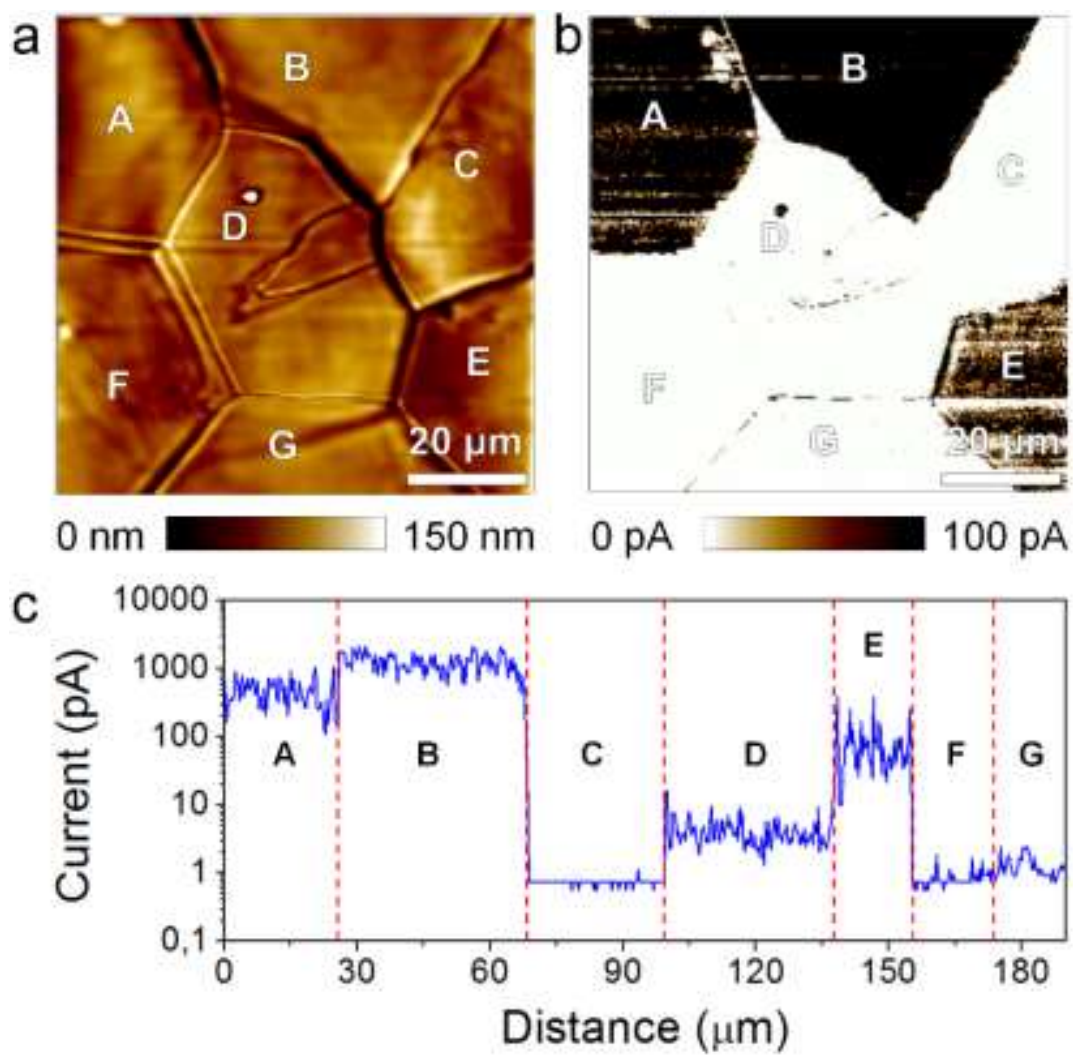

Figure 3. CAFM Characterization of as-grown $h$-BN/Pt. (a) Topographic and (b) current maps simultaneously collected on $h$-BN grown on a polycrystalline Pt substrate, under a bias of $-2 \mathrm{~V}$ (applied to the substrate, tip grounded). (c) Assembly of cross sections collected at the different grains of the current map in (b). 

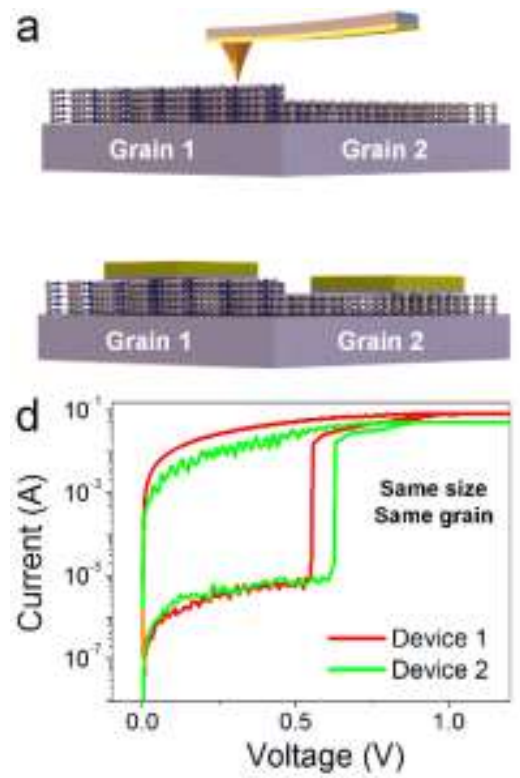
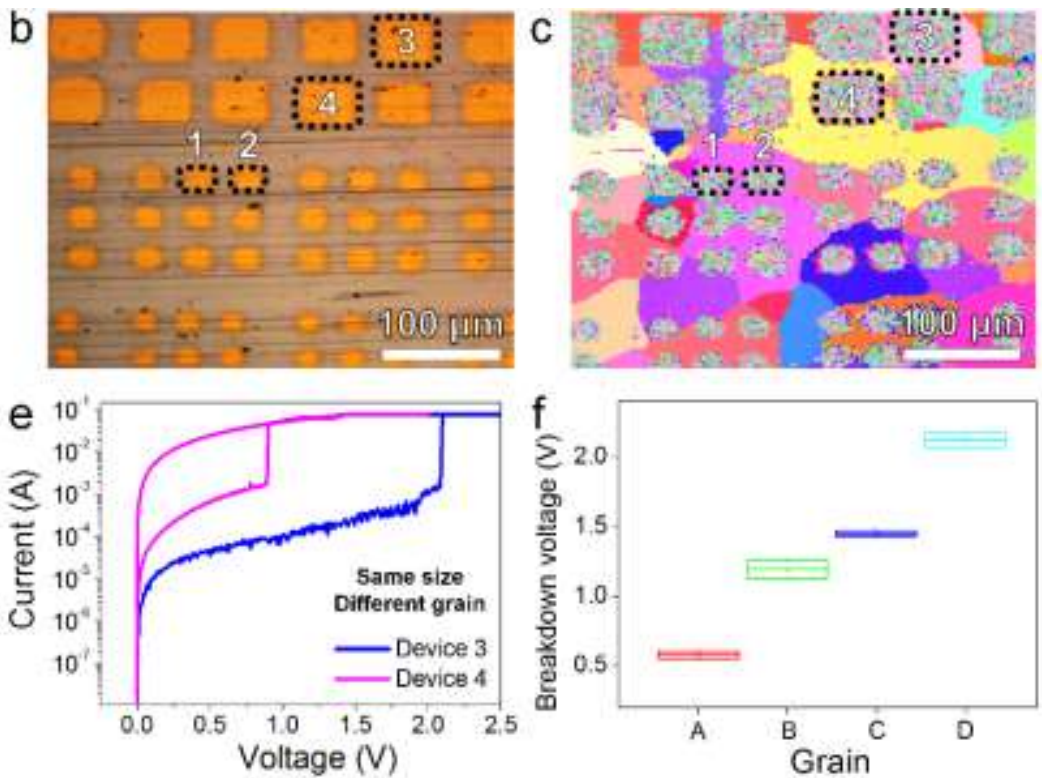

Figure 4. Device level characterization of $h$-BN stacks. (a) Schematic of as-grown $h$-BN/Pt (top) and $h$-BN based devices (bottom). The device cell is an $\mathrm{Au} / \mathrm{Ti} / h$-BN/Pt structure. (b) Optical image and (c) EBSD map of matrixes of $\mathrm{Au} / \mathrm{Ti} / h-\mathrm{BN} / \mathrm{Pt}$ cells with different lateral sizes. As this map has been collected with the presence of $h$ - $\mathrm{BN}$, it is only meaningful to distinguish different grains, not to assess the real crystallographic orientation of each grain. For this reason, the color scale has been intentionally removed. (d) I-V curves collected on two devices with the same size $(25 \mu \mathrm{m} \times 25 \mu \mathrm{m})$ within the same Pt grain. (e) I-V curves collected on two devices with the same size $(50 \mu \mathrm{m} \times 50 \mu \mathrm{m})$ located at different Pt grains. (f) Analysis of the BD voltage for $\mathrm{Au} / \mathrm{Ti} / h$ BN/Pt devices patterned on 4 different Pt grains (located outside the region of 4c). 


\section{Electrical homogeneity of chemical vapor deposited hexagonal boron nitride}

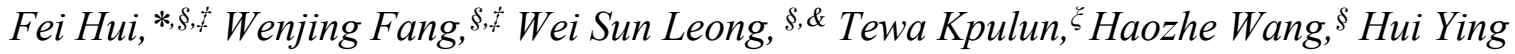
Yang, ${ }^{\&}$ Marco A. Villena*, Gary Harris, ${ }^{\xi}$ Jing Kong, ${ }^{\S}$ Mario Lanza*

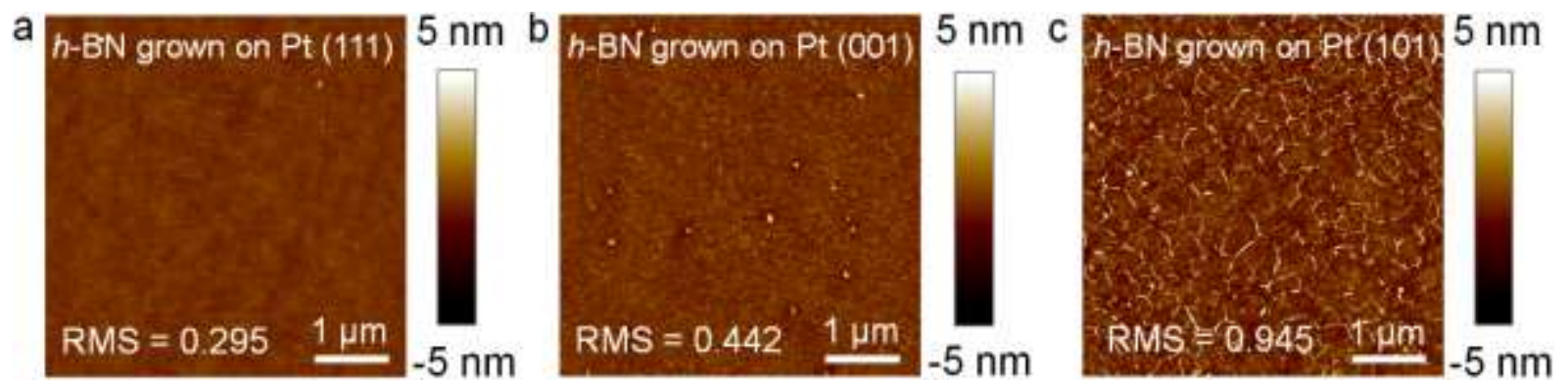

Figure S1. AFM characterization of the $h-\mathrm{BN} / 300-\mathrm{nm}-\mathrm{SiO}_{2} / \mathrm{Si}$ sample displayed in Figure 2c. The $h$-BN has been grown on via CVD on a polycrystalline Pt substrate and transferred on a 300$\mathrm{nm}-\mathrm{SiO}_{2} / \mathrm{Si}$ sample. After the transfer, the optical microscope photograph (Figure 2c) shows shapes perfectly matching the Pt grains observed via EBSD (Figure 2b). Therefore, it is possible to know the crystallographic orientation of the Pt substrate on which the $h$-BN was grown. Panels (a)-(c) in Figure S1 show that the roughness and density of wrinkles in the $h$-BN strongly depend on the crystallographic orientation of the Pt substrate on which it was grown.

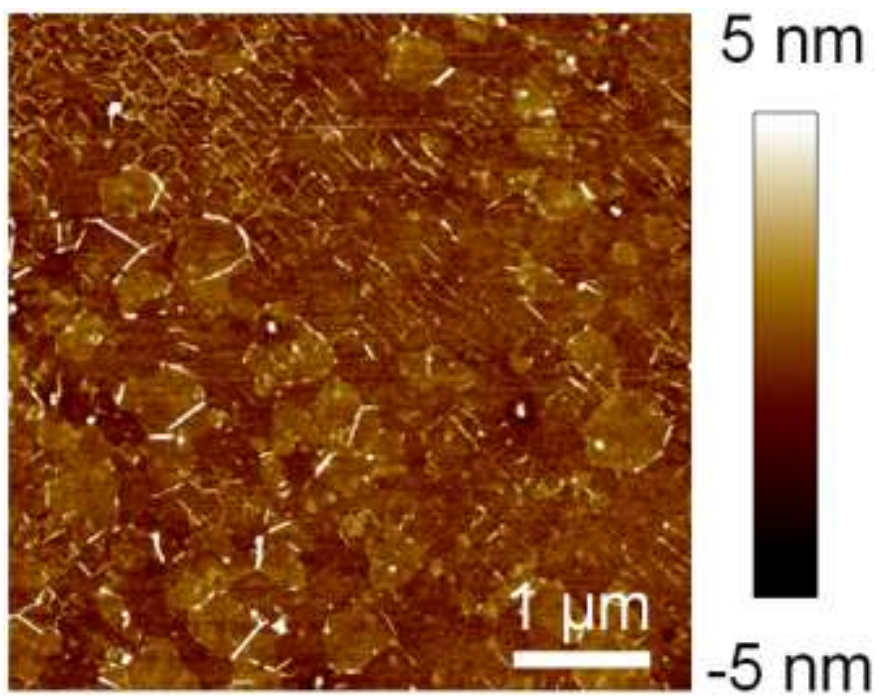

Figure S2. AFM characterization of a $h-\mathrm{BN} / 300-\mathrm{nm}-\mathrm{SiO}_{2} / \mathrm{Si}$ sample, on a region on which the h-BN was previously grown on $\operatorname{Pt}(101)$. This is Zoom-in image of Figure S1c. This image shows multilayer $h$-BN islands. 

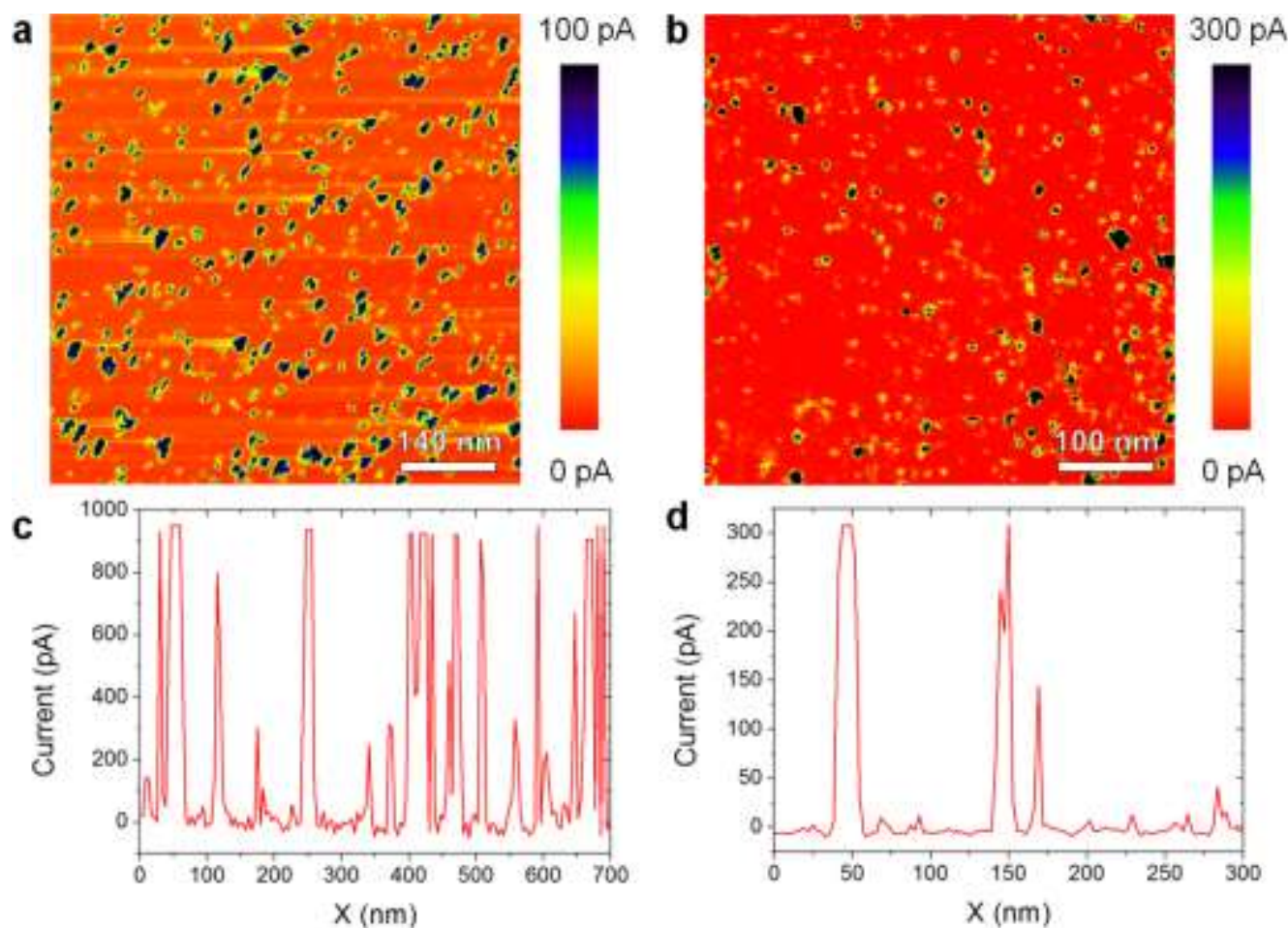

Figure S3. Current maps collected with the tip of the CAFM on the surface of (a) $4 \mathrm{~nm}$ thick $\mathrm{HfO}_{2}$ and (b) $2 \mathrm{~nm}$ thick $\mathrm{TiO}_{2}$ films, both of them grown via atomic layer deposition on a conductive substrate $\left(\mathrm{Zr}\right.$ below the $\mathrm{HfO}_{2}$ and $\mathrm{n}++\mathrm{Si}$ below the $\left.\mathrm{TiO}_{2}\right)$. The current fluctuations are 2-3 orders of magnitude. (c) and (d) show the cross sections of the maps in (a) and (b) respectively.

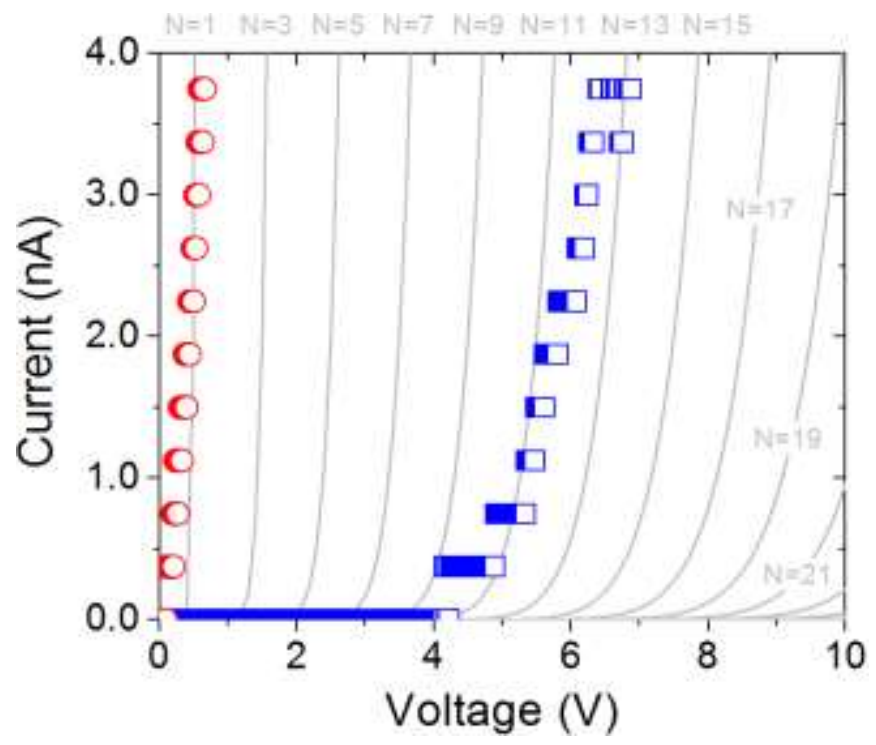

Figure S4. Calculation of FNT conduction for different thicknesses ( $\mathrm{N}$ is the number of layers). Typical experimental IV curves measured on grain B (red symbols) and grain D (blue symbols) of Figure $3 b$ of the manuscript. 


\begin{tabular}{ccc}
\hline Grain & $\begin{array}{c}\text { Current } \\
\text { (for a voltage of -1 V) }\end{array}$ & $\begin{array}{c}\text { Voltage } \\
\text { (for a current of 5 pA) }\end{array}$ \\
\hline A & $5 \mathrm{nA}$ & $0.5 \mathrm{~V}$ \\
$\mathrm{~B}$ & $5 \mathrm{nA}$ & $0.1 \mathrm{~V}$ \\
$\mathrm{C}$ & $<1 \mathrm{pA}$ & $6 \mathrm{~V}$ \\
$\mathrm{D}$ & $<1 \mathrm{pA}$ & $2 \mathrm{~V}$ \\
$\mathrm{E}$ & $<1 \mathrm{pA}$ & $1 \mathrm{~V}$ \\
$\mathrm{~F}$ & $<1 \mathrm{pA}$ & $5 \mathrm{~V}$ \\
$\mathrm{G}$ & $<1 \mathrm{pA}$ & $5 \mathrm{~V}$ \\
\hline
\end{tabular}

Table S1 Electrical measurements are conducted on each grain with the constant voltage and constant 5 pA, respectively. 